Rosa del Rocío Pinargote Chancay; Yasmin Casrtillo Merino; Bryan Ramón Figueroa Merino; Geomayra Betzabeth Cedeño Chavarrria

http://dx.doi.org/10.35381/s.v.v3i6.484

\title{
Aspectos socio-demográficos del paciente con tuberculosis (tb)
}

Socio-demographical aspects of the patient with tuberculosis (tb)

\author{
Rosa del Rocío Pinargote Chancay \\ rosa.pinargote@unesum.edu.ec \\ Universidad Estatal del Sur de Manabí, Jipijapa \\ Ecuador \\ https://orcid.org/0000-0001-9899-9243 \\ Yasmin Castillo Merino \\ yascasme@hotmail.es \\ Universidad Estatal del Sur de Manabí, Jipijapa \\ Ecuador \\ https://orcid.org/0000-0002-1442-1725 \\ Bryan Ramón Figueroa Merino \\ brymen@hotmail.com \\ Centro de salud tipo A Simón Bolívar la Sexta; Quinindé \\ Ecuador \\ https://orcid.org/0000-0002-7369-7369 \\ Geomayra Betzabeth Cedeño Chavarria. \\ geomayra1992@hotmail.es \\ Centro de salud Jipijapa, Jipijapa \\ Ecuador \\ https://orcid.org/0000-0001-6728-4341
}

Recibido: 15 de mayo de 2019

Aprobado: 14 de Junio de 2019

\section{RESUMEN}

En el presente artículo se caracterizaron los aspectos socio- demográficos del paciente TB en el Cantón de Manabí del Ecuador. Para ello, se llevó a cabo un estudio de tipo observacional, descriptivo, transversal y prospectivo. La población la constituyeron 40 pacientes que asisten a consultas de controles en los centros de salud que atienden pacientes con TB en el Canton de Manabí del Ecuador. El instrumento utilizado fue un 
Rosa del Rocío Pinargote Chancay; Yasmin Casrtillo Merino; Bryan Ramón Figueroa Merino; Geomayra Betzabeth Cedeño Chavarrria

cuestionario y la técnica la entrevista estructurada. De sus resultados, se puede indicar que el $75 \%$ de las opiniones favorecieron el factor afectivo y emocional de las familias. En sus conclusiones se destaca el hecho en la cual un alto porcentaje de los pacientes con TB investigados presenta un nivel socioeconómico por debajo de la media nacional y reciben un alto apoyo familiar en relación al afecto y la parte emocional.

Descriptors: Socio-demográficos; Familia; Paciente TB; Sexo y edad.

\begin{abstract}
This article characterized the socio-demographic aspects of the TB patient in the canton of Manabí of Ecuador. To this end, an observational, descriptive, cross-sectional and prospective study was carried out. The population was made up of 40 patients attending check-ups at health centers serving TB patients in the Canton of Esmeralda, Ecuador. The instrument used was a questionnaire and the technique structured interview. From their results, it can be noted that $75 \%$ of the opinions favored the affective and emotional factor of the families. Its findings highlight the fact that a high percentage of patients with researched TB have a socioeconomic level below the national average and receive high family support in relation to affection and the emotional side.
\end{abstract}

Descriptors: Socio-demographic; Family; TB patient; Sex and age.

\title{
INTRODUCCIÓN
}

La Organización Mundial de la Salud (OMS) informa que un tercio de la población mundial está infectada por Mycobacterium tuberculosis; cada año se estima una ocurrencia cercana a ocho millones de casos nuevos y 1.5 millones de defunciones por tuberculosis pulmonar (Gómez y col, 2005). En Ecuador, el estimado por la Organización Mundial de la Salud, fue de 8.400 casos nuevos de TB (51.6/100 mil habitantes) (7), incluyendo aquellos con coinfección TB/VIH. Sin embargo, Sistema Nacional de Salud (SNS) que comprende la Red Pública Integral de Salud (RPIS) y la Red Prevención, diagnóstico, tratamiento y control de la tuberculosis / Guía de Práctica Clínica 18 Complementaria (2 018) diagnosticó y notificó 5.215 casos (32.03/100 mil habitantes) cumpliendo el $62.08 \%$ de lo estimado. De los casos notificados 5.097 corresponden a casos nuevos y recaídas, 
Rosa del Rocío Pinargote Chancay; Yasmin Casrtillo Merino; Bryan Ramón Figueroa Merino; Geomayra Betzabeth Cedeño Chavarrria

y 118 casos previamente tratados. En coinfección TB/VIH se notificaron 545 casos, que representan el 10,45 \% de los casos TB. En TB MDR/RR se estimaron 370 casos e iniciaron tratamiento 135 casos, que representan el 36.48\%. Según la cohorte de 2014, el tratamiento exitoso en el país para los casos nuevos y recaídas fue del $76,87 \%$ (Baldeon, 2007). La tasa de mortalidad reportada por el Instituto Nacional de Estadística y Censos (INEC, 2015), es de 2,59/100 mil habitantes en 2014. Considerando los cambios estratégicos en el abordaje de la salud pública y de la atención primaria de la salud, la prevención y el control de la tuberculosis en Ecuador genera un cambio en la parte organizacional y en la prestación de servicios con enfoque en grupos vulnerables y/o con factores de riesgo acorde a los ciclos de vida. Ante esta posición, en el artículo se caracterizaron los aspectos socio- demográficos del paciente TB en el cantón de Manabí del Ecuador.

\section{DESARROLLO}

La tuberculosis (TB) continúa siendo un problema de salud pública, según datos de la Organización Mundial de la Salud (2018), se estimó a nivel mundial 10.4 millones casos nuevos en todas sus formas y se notificaron 6.1 millones. Ante ello, la actividad central de la atención integral del paciente TB, permite cortar la cadena epidemiológica disminuyendo los focos infecciosos, aliviando a la vez el sufrimiento humano que ocasiona la enfermedad y mejorando su calidad de vida. El enfoque de atención del Paciente con Tuberculosis permite constituir un proceso de intervención multidisciplinario. La adherencia del paciente al tratamiento depende en gran parte de la relación establecida entre el paciente, el personal de salud que lo atiende y el establecimiento de salud. En el caso de que la administración del tratamiento sea apoyado por un agente comunitario de salud, debe mantenerse la confidencialidad y una buena relación con el paciente. (Ministerio de Salud Pública del Ecuador, 2016)

Cabe destacar, que la tuberculosis en el Ecuador tiene una tendencia estable, sin embargo existe una importante brecha entre las tasas de prevalencia y tasa de 
Rosa del Rocío Pinargote Chancay; Yasmin Casrtillo Merino; Bryan Ramón Figueroa Merino; Geomayra Betzabeth Cedeño Chavarrria

incidencia de la tuberculosis, en comparación a las tasas estimadas por la Organización Mundial de la Salud, alrededor de 80 y 40 × 100000 respectivamente. Esta brecha, que podrían interpretarse de dos maneras: una que la tasa de la OMS está sobreestimada y otra que no se están detectando los casos de tuberculosis, en especial los casos extra pulmonares.

\section{El apoyo familiar en el paciente TB}

El apoyo familiar ha sido un constructo poco estudiado, por tanto carece de una definición unificada, pero se caracteriza por abarcar varias dimensiones entre las cuales se pueden mencionar la comunicación, conflictos intrafamiliares, resolución de problemas, grado de afectividad y buen planteamiento de reglas.

El apoyo familiar, definido como proceso psicosocial, implica considerar que las formas en las que éste se dé y los significados y valores que se le atribuyan, sólo podrán ser comprendidos a la luz de un contexto socio histórico específico. Esto significa que el apoyo familiar, además de ser una estrategia individual y relacional particular, anclada a su vez en condiciones biológicas, se configura y se transforma de manera específica de acuerdo con las condiciones sociales e históricas presentes. (Estrada y col, 1997)

\section{Tipos de apoyo familiar}

Según Cadena ( 2016), el apoyo familiar es considerado como la forma potencial de apoyo y capacidad para tener influencia en la salud y es de manera empírica. La importancia de un tipo u otro dependerá en cada caso de la relevancia de la fuente de apoyo para la persona y del problema concreto que requiera tal apoyo, en este sentido menciona como tipos de apoyo, los siguientes:

Apoyo afectivo.- Es la capacidad de reacción que presenta el familiar con manifestaciones de sentimientos y emociones; en un marco interactivo, es decir, dar afecto al paciente con tuberculosis pulmonar, ayudándole siempre con motivación, preocupación, tranquilidad, dándole aliento y ánimo. 
Rosa del Rocío Pinargote Chancay; Yasmin Casrtillo Merino; Bryan Ramón Figueroa Merino; Geomayra Betzabeth Cedeño Chavarrria

Apoyo emocional.- Comprende la empatía, el cuidado, el amor, la confianza y parece ser uno de los tipos de apoyo más importantes. Este tipo de apoyo está recogido, de una u otra manera, en las concepciones de apoyo antes mencionadas. En general, cuando las personas consideran a otra persona que ofrece apoyo, se tiende a conceptualizar en torno al apoyo emocional. Otros tipos de apoyo social suelen ser menos utilizados en la explicación y estudio de este concepto pero en ciertas situaciones suelen tener una gran importancia.

Apoyo instrumental.- Se ofrece cuando se emiten conductas instrumentales que directamente ayudan a quien lo necesita; se ayuda a otro a hacer o acabar el trabajo, se cuida de alguien. Es evidente que todo acto puramente instrumental tiene también consecuencias psicológicas (así, la ayuda en el trabajo puede ser una ayuda o una fuente de información y valoración, y no siempre positiva; necesitas ayuda y no eres autosuficiente, no tienes capacidad).

\section{Adherencia del tratamiento en pacientes TB}

La adherencia al tratamiento de la tuberculosis es uno de los elementos claves para la curación de los enfermos (Puc-Franco y col, 2012). Actualmente, uno de los obstáculos para el control de la tuberculosis es el abandono del tratamiento. La OMS considera como abandono el incumplimiento por el paciente del régimen fármaco- terapéutico por un período mayor de dos meses. Algunos autores lo definen como la inasistencia continua del paciente a la unidad de salud por un período mayor de 29 días; otros, como la inasistencia a los servicios de salud por tiempo indefinido, independientemente del tiempo que dure el tratamiento (USAID, 2000). El abandono de tratamiento de la tuberculosis es uno de los factores más importantes que disminuye la eficiencia de la terapia, asociándose a fracasos, mayor mortalidad, desarrollo de resistencia bacteriana y un período de contagiosidad (Herrera, 2011).

\section{MATERIALES Y MÉTODOS}


Rosa del Rocío Pinargote Chancay; Yasmin Casrtillo Merino; Bryan Ramón Figueroa Merino; Geomayra Betzabeth Cedeño Chavarrria

El estudio fue de tipo observacional, descriptivo, transversal, prospectivo, realizado en los centros de salud que atienden pacientes con TB en el Canton de Esmeralda, Ecuador. La población la constituyeron 40 pacientes que asisten a consultas de controles. El instrumento utilizado fue un cuestionario contentivo de los datos sociales y demográficos que distinguen a cada paciente. La técnica utilizada fue la entrevista estructurada.

\section{RESULTADOS}

Los resultados de la información aportada por los pacientes investigados se presentan a continuación en tablas con sus respectivos gráficos.

Análisis descriptivo frecuencial del indicador sexo del paciente TB del Cantón de Manabí del Ecuador. Tabla 1.

\begin{tabular}{|l|l|l|l|l|}
\hline $\begin{array}{l}\text { Análisis/ } \\
\text { indicador }\end{array}$ & Fr & $\%$ & FrA & $\%$ A \\
\hline Femenino & 17 & 42 & 17 & 42 \\
\hline Masculino & 23 & 58 & 40 & 100 \\
\hline Total & 40 & 100 & 40 & 100 \\
\hline
\end{tabular}

Fuente: Elaboración propia (2019).

Análisis descriptivo frecuencial del indicador sexo del paciente diabético en el contexto rural de la provincia de Manabí, Ecuador.

Grafico 1.

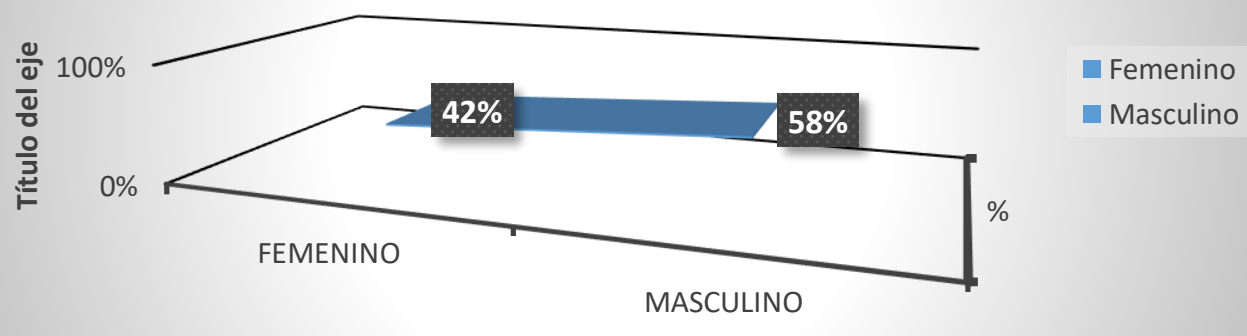


Rosa del Rocío Pinargote Chancay; Yasmin Casrtillo Merino; Bryan Ramón Figueroa Merino; Geomayra Betzabeth Cedeño Chavarrria

Fuente: Elaboración propia (2019).

En la tabla y grafico 1, se presenta el análisis del indicador sexo en pacientes con TB. En la misma se observa un predominio del sexo masculino con el $58 \%$ de los casos y 17 del sexo femenino que representa el $42 \%$ del total de los pacientes investigados.

Análisis descriptivo frecuencial del indicador edad del paciente con TB del Cantón de Manabí del Ecuador.

Tabla 2.

\begin{tabular}{|l|l|l|l|l|}
\hline $\begin{array}{l}\text { Análisis/ } \\
\text { indicador }\end{array}$ & $\mathbf{F r}$ & $\mathbf{F r A}$ & \%A \\
\hline$<=20$ años & $\mathbf{2}$ & $\mathbf{5}$ & $\mathbf{2}$ & $\mathbf{5}$ \\
\hline $21-40$ años & $\mathbf{1 4}$ & $\mathbf{3 5}$ & $\mathbf{1 6}$ & $\mathbf{4 0}$ \\
\hline $41-60$ años & $\mathbf{1 8}$ & $\mathbf{4 5}$ & $\mathbf{3 4}$ & $\mathbf{8 5}$ \\
\hline $61-80$ años & $\mathbf{5}$ & $\mathbf{1 3}$ & $\mathbf{3 9}$ & $\mathbf{9 8}$ \\
\hline$>=81$ años & $\mathbf{1}$ & $\mathbf{2}$ & $\mathbf{4 0}$ & $\mathbf{1 0 0}$ \\
\hline Total & $\mathbf{4 0}$ & $\mathbf{1 0 0}$ & $\mathbf{4 0}$ & $\mathbf{1 0 0}$ \\
\hline
\end{tabular}

Fuente: Elaboración propia (2019).

Análisis descriptivo frecuencial del indicador edad del paciente con TB del Cantón de Manabí del Ecuador.

Grafico 2. 
Rosa del Rocío Pinargote Chancay; Yasmin Casrtillo Merino; Bryan Ramón Figueroa Merino; Geomayra Betzabeth Cedeño Chavarrria

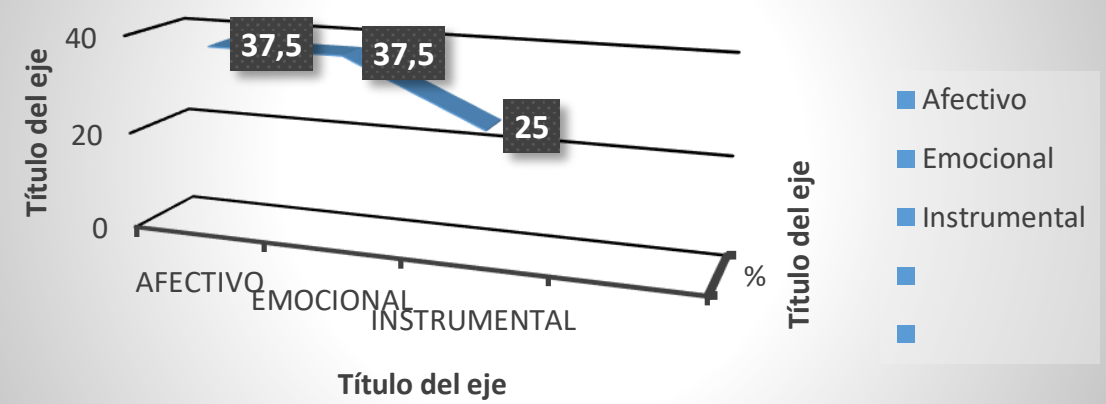

Fuente: Elaboración propia (2019).

En la tabla y grafico 2, se presenta el análisis del indicador edad en pacientes con TB. En la misma se indica que el $45 \%$ del total de los pacientes investigados manifestó tener entre 41 60 años de edad. Seguido del 35\% y 13\% que se ubicaron en edades de $21-40$ y $61-80$ años de edad respectivamente. Por otro lado, solo un paciente que representa el $2 \%$ de los investigados era mayor a 81 años de edad.

Análisis descriptivo frecuencial del indicador nivel socio económico del paciente con TB del Cantón de Manabí del Ecuador.

Tabla 3.

\begin{tabular}{|l|l|l|l|l|}
\hline $\begin{array}{l}\text { Análisis/ } \\
\text { indicador }\end{array}$ & Fr & $\%$ & FrA & \%A \\
\hline Muy Alto & $\mathbf{0}$ & $\mathbf{0}$ & $\mathbf{0}$ & $\mathbf{0}$ \\
\hline Alto & $\mathbf{2}$ & $\mathbf{5}$ & $\mathbf{2}$ & $\mathbf{5}$ \\
\hline Medio & $\mathbf{1 0}$ & $\mathbf{2 5}$ & $\mathbf{1 2}$ & $\mathbf{3 0}$ \\
\hline Bajo & $\mathbf{1 5}$ & $\mathbf{3 7}$ & $\mathbf{2 7}$ & $\mathbf{6 7}$ \\
\hline Muy bajo & $\mathbf{1 3}$ & $\mathbf{3 3}$ & $\mathbf{4 0}$ & $\mathbf{1 0 0}$ \\
\hline Total & $\mathbf{4 0}$ & $\mathbf{1 0 0}$ & $\mathbf{4 0}$ & $\mathbf{1 0 0}$ \\
\hline
\end{tabular}

Fuente: Elaboración propia (2019).

Análisis descriptivo frecuencial del indicador nivel socio económico del paciente con TB del Cantón de Manabí del Ecuador. 
Rosa del Rocío Pinargote Chancay; Yasmin Casrtillo Merino; Bryan Ramón Figueroa Merino; Geomayra Betzabeth Cedeño Chavarrria

\section{Grafico 3.}
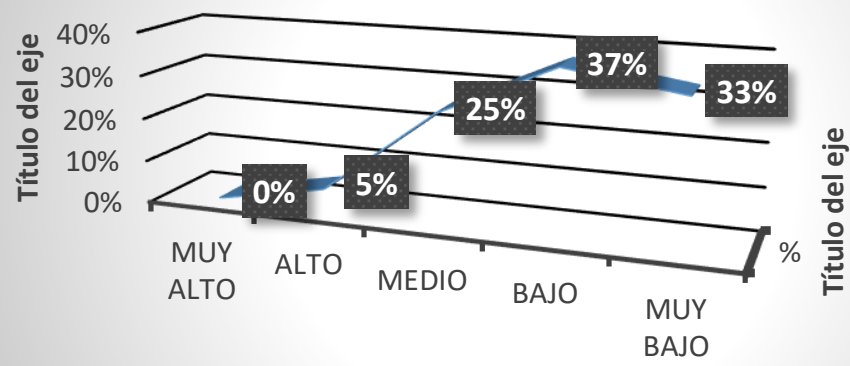

Muy Alto

Alto

Medio

Bajo

Muy bajo

Título del eje

Fuente: Elaboración propia (2019).

En la tabla y grafico 3, se presenta el análisis del indicador nivel socioeconómico del paciente TB del Cantón de Manabí del Ecuador. En la misma se indica que el 37\% de los pacientes manifestaron pertenecer a un nivel socio económico bajo y el $33 \%$ a un nivel muy bajo. Los menores porcentajes con el $25 \%$ y $5 \%$ indicaron pertenecer a un nivel medio y alto respectivamente. Se destaca el hecho, en la cual el nivel socio económico muy alto no presento opinión alguna entre los investigados.

Análisis descriptivo frecuencial del indicador reincidencia de la enfermedad en pacientes con TB del Cantón de Manabí del Ecuador.

Tabla 4.

\begin{tabular}{|l|l|l|l|l|}
\hline $\begin{array}{l}\text { Análisis/ } \\
\text { indicador }\end{array}$ & Fr & $\%$ & FrA & $\% A$ \\
\hline $\mathrm{Si}$ & $\mathbf{1 2}$ & $\mathbf{3 0}$ & $\mathbf{1 2}$ & $\mathbf{3 0}$ \\
\hline No & $\mathbf{2 8}$ & $\mathbf{7 0}$ & $\mathbf{4 0}$ & $\mathbf{1 0 0}$ \\
\hline Total & $\mathbf{4 0}$ & $\mathbf{1 0 0}$ & $\mathbf{4 0}$ & $\mathbf{1 0 0}$ \\
\hline
\end{tabular}

Fuente: Elaboración propia (2019).

Análisis descriptivo frecuencial del indicador reincidencia de la enfermedad en pacientes con TB del Cantón de Manabí del Ecuador.

Grafico 4. 
Rosa del Rocío Pinargote Chancay; Yasmin Casrtillo Merino; Bryan Ramón Figueroa Merino; Geomayra Betzabeth Cedeño Chavarrria

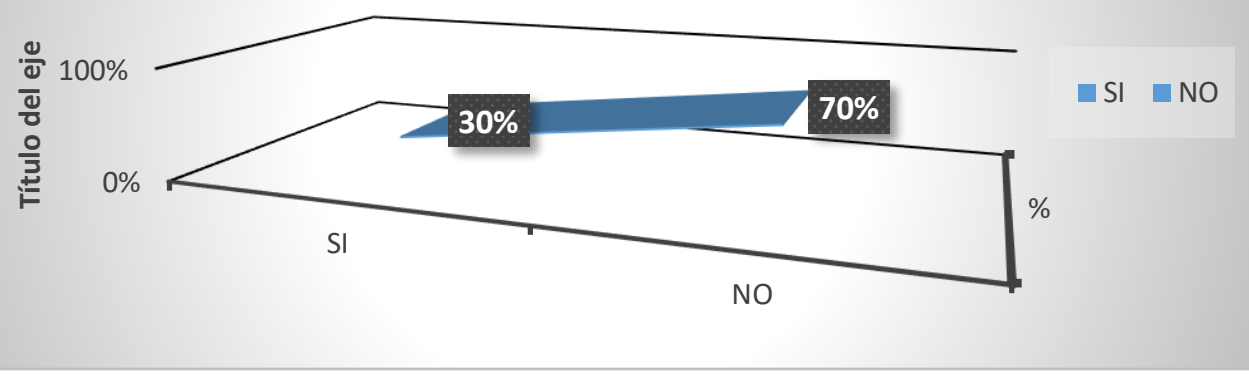

Fuente: Elaboración propia (2019).

En la tabla y grafico 4 , se presenta el análisis del indicador reincidencia de la enfermedad en pacientes con TB del Cantón de Manabí del Ecuador. En la misma se señala que el $70 \%$ de los pacientes que asisten a los centros de salud de Manabí a control de la enfermedad no son reincidentes, mientras que el $30 \%$ manifestó en forma positiva.

Análisis descriptivo frecuencial de los tipos de apoyo familiar que afectaron a los pacientes con diagnóstico de TB del Cantón de Manabí del Ecuador.

Tabla 5.

\begin{tabular}{|l|l|l|l|l|}
\hline $\begin{array}{l}\text { Análisis/ } \\
\text { indicador }\end{array}$ & Fr & $\%$ & FrA & \%A \\
\hline Afectivo & 15 & $\mathbf{3 7 , 5}$ & $\mathbf{1 5}$ & $\mathbf{3 7 , 5}$ \\
\hline Emocional & 15 & $\mathbf{3 7 , 5}$ & $\mathbf{3 0}$ & $\mathbf{7 5}$ \\
\hline Instrumental & 10 & $\mathbf{2 5}$ & $\mathbf{4 0}$ & $\mathbf{1 0 0}$ \\
\hline Total & $\mathbf{4 0}$ & $\mathbf{1 0 0}$ & $\mathbf{4 0}$ & $\mathbf{1 0 0}$ \\
\hline
\end{tabular}

Análisis descriptivo frecuencial de los tipos de apoyo familiar que afectaron a los pacientes con diagnóstico de TB del Cantón de Manabí del Ecuador.

Grafico 5. 
Rosa del Rocío Pinargote Chancay; Yasmin Casrtillo Merino; Bryan Ramón Figueroa Merino; Geomayra Betzabeth Cedeño Chavarrria

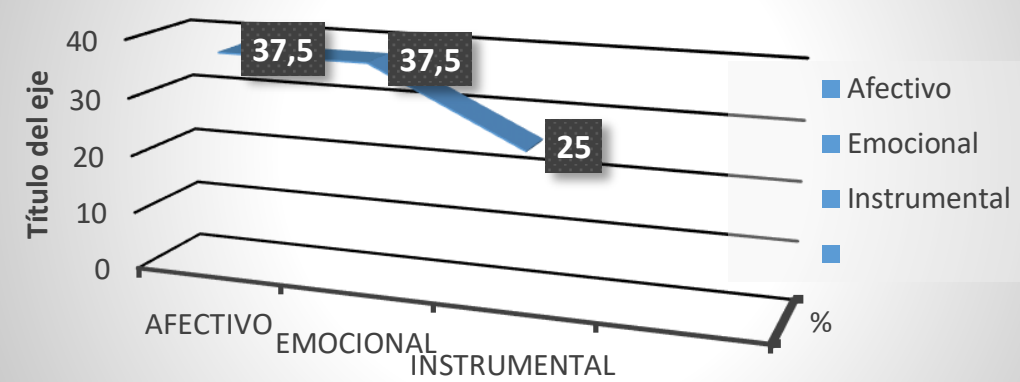

Fuente: Elaboración propia (2019).

En la tabla y grafico 5, se presenta el análisis descriptivo de los tipos de apoyo familiar que afectaron a los pacientes con diagnóstico de TB del Cantón de Manabí del Ecuador. Se observa entre sus resultados que el $75 \%$ de las opiniones se manifestaron en opinión favorecida hacia el factor afectivo y emocional. El menor porcentaje se indicó para el factor apoyo instrumental con solo el $25 \%$ de las opiniones.

Análisis descriptivo frecuencial de los aspectos sociales que afectan a los pacientes con diagnóstico de TB del Cantón de Manabí del Ecuador.

Tabla 6.

\begin{tabular}{|l|l|l|l|l|}
\hline $\begin{array}{l}\text { Análisis/ } \\
\text { indicador }\end{array}$ & Fr & $\%$ & FrA & $\% \mathbf{A}$ \\
\hline $\begin{array}{l}\text { La vada } \\
\text { personal }\end{array}$ & 20 & $\mathbf{5 0}$ & $\mathbf{2 0}$ & $\mathbf{5 0}$ \\
\hline $\begin{array}{l}\text { El abandono } \\
\text { de la familia }\end{array}$ & 2 & $\mathbf{5}$ & $\mathbf{2 2}$ & $\mathbf{5 5}$ \\
\hline $\begin{array}{l}\text { La relación en } \\
\text { pareja }\end{array}$ & 6 & $\mathbf{1 5}$ & $\mathbf{2 8}$ & $\mathbf{7 0}$ \\
\hline El rechazo soci & $\mathbf{1 2}$ & $\mathbf{3 0}$ & $\mathbf{4 0}$ & $\mathbf{1 0 0}$ \\
\hline Total & $\mathbf{4 0}$ & $\mathbf{1 0 0}$ & $\mathbf{4 0}$ & $\mathbf{1 0 0}$ \\
\hline
\end{tabular}

Fuente: Elaboración propia (2019). 
Rosa del Rocío Pinargote Chancay; Yasmin Casrtillo Merino; Bryan Ramón Figueroa Merino; Geomayra Betzabeth Cedeño Chavarrria

Análisis descriptivo frecuencial de los aspectos de tipo familiar y social que afectaron a los pacientes con diagnóstico de Tв del Cantón de Manabí del Ecuador.

Grafico 6.

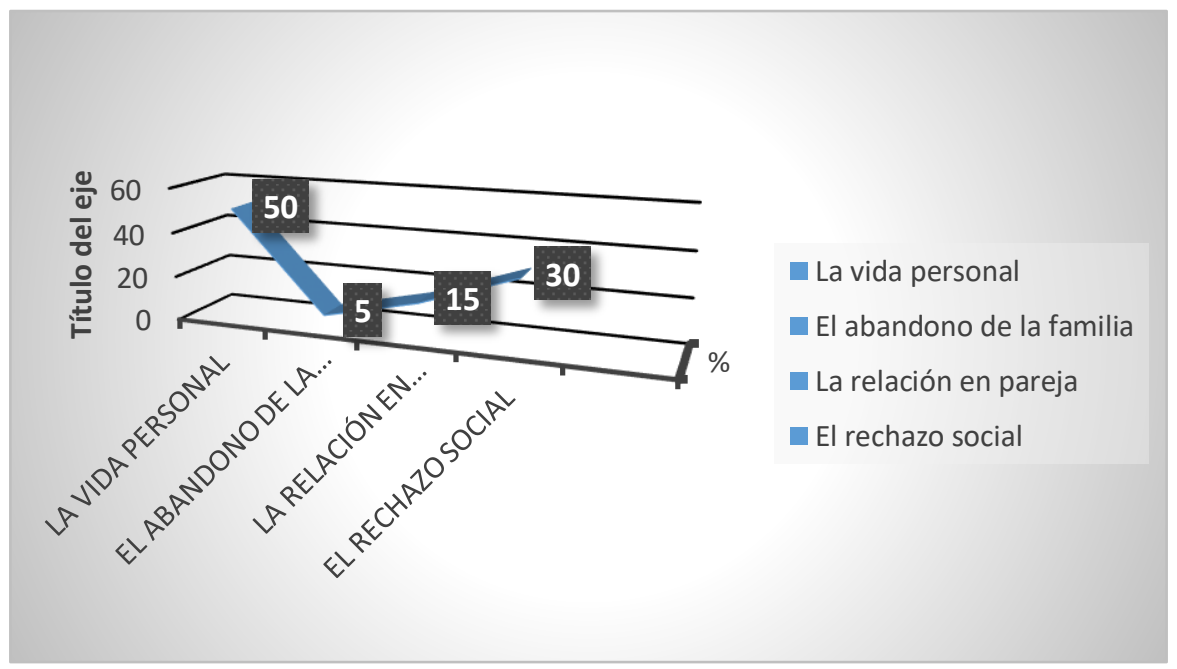

Fuente: Elaboración propia (2019).

En la tabla y grafico 6 , se presentan los aspectos familiares y sociales que afectaron a los pacientes con diagnostico TB del Cantón de Manabí del Ecuador, en la misma se observa que la mitad de ellos representados por 20 de los cuarentas pacientes manifestaron que la enfermedad afecto su vida personal, seguido del $30 \%$ que indicaron el rechazo social. Por otro lado, se indica que el $15 \%$ perdieron la relación que llevaban en parejas. El menor porcentaje se indicó para el rechazo familiar con solo el $5 \%$ del total de las opiniones.

\section{CONCLUSIONES}

-Se indica un predominio del sexo masculino sobre la enfermedad y cuya menor edad de aparición fue a los 17 años y el mayor investigado se correspondía a un paciente mayor de 81 años.

-Se evidencio que un alto porcentaje de los pacientes con TB investigados presenta un nivel socioeconómico por debajo de la media nacional.

- Se constató un alto apoyo familiar en relación al afecto hacia al paciente con tuberculosis pulmonar, ayudándole siempre con motivación, preocupación, tranquilidad y dándole aliento y ánimo, de igual forma reciben cuidado, amor y confianza. 
Arbitrada Interdisciplinaria de Ciencias de la Salud. SALUD Y VIDA

Volumen 3. Número 6. Año 3. Julio - Diciembre 2019

Hecho el depósito de Ley: FA2016000010

ISSN: 2610-8038

FUNDACIÓN KOINONIA (F.K).

Santa Ana de Coro, Venezuela.

Rosa del Rocío Pinargote Chancay; Yasmin Casrtillo Merino; Bryan Ramón Figueroa Merino; Geomayra Betzabeth Cedeño Chavarrria

-La sensibilidad de la enfermedad fue de un 7,02 \%, en la que se indica un valor por debajo de la media nacional del Ecuador, la cual está situada en 15 pacientes por cada 100 mil habitantes.

-Asimismo, se evidenció un alto porcentaje de disfunción familiar y rechazo social, manifiesto por los pacientes investigados, como la principal causa del abandono del tratamiento.

\section{REFERENCIAS CONSULTADAS}

1. Baldeon, M. (2007). Relación entre el Clima Social Familiar y Nivel de Autoestima de los Pacientes con TBC. Tesis para Obtener el titulo de Lic. en enfermeria. Lima - Peru.

2. Estrada, M y Feliu E (1997). Modelo de atencion de enfermeria comunitaria. Primera ed. Cuba: WALSUP.

3. Gómez-Camelo, D. (2005). Análisis comparado de los sistemas de salud de la región Andina y el Caribe. Revista de Salud Pública, 7, (3), pp. 305-316.

4. Guía de Práctica Clínica (2018). Prevención, diagnóstico, tratamiento y control de la tuberculosis .Segunda edición

5. Herrera, M (2011). Perfil del paciente con tuberculosis que abandona el tratamiento en Chile Zulema. II . (12-15).

6. INEC (2015). Estadísticas de Nacimientos y Defunciones del Ecuador.

7. Ministerio de Salud Pública del Ecuador (2016) Normas y Procedimiento para la Atención de la Salud Reproductiva MSP.

8. OMS (2018). Organización Mundial de la Salud. Tuberculosis. [Online].; 2018 [cited 2018 Septiembre 28. Available from: http://www.who.int/es/news-room/factsheets/detail/tuberculosis

9. Puc-Franco M, Caamal-Ley A y Vargas-González A.(2012). Determinantes sociales relacionados con el tratamiento de tuberculosis. Rev Biomed. 2012 Febrero; 23(113-120). 
Rosa del Rocío Pinargote Chancay; Yasmin Casrtillo Merino; Bryan Ramón Figueroa Merino; Geomayra Betzabeth Cedeño Chavarrria

10. USAID (2000). Agencia de los Estados Unidos para el Desarrollo Internacional y Ministerio de Salud. Vivir la tuberculosis. In Estudio socio antropologico de la TBC en las zonas de alto riesgo. San Cosme, El Agustino; 2000. p. 29.

\section{REFERENCES CONSULTED}

1. Baldeon, M. (2007). Relationship between Family Social Climate and Level of SelfEsteem of Patients with TB. Thesis to obtain the title of Nursing Degree. Lima Peru.

2. Estrada, M and Feliu E (1997). Community nursing care model. First ed. Cuba: WALSUP.

3. Gómez-Camelo, D. (2005). Comparative analysis of health systems in the Andean region and the Caribbean. Public Health Magazine, 7, (3), pp. 305-316.

4. Clinical Practice Guide (2018). Prevention, diagnosis, treatment and control of tuberculosis. Second edition

5. Herrera, M (2011). Profile of the patient with tuberculosis who abandons treatment in Chile Zulema. II. (12-15).

6. INEC (2015). Birth and Death Statistics of Ecuador.

7. Ministry of Public Health of Ecuador (2016) Rules and Procedure for Reproductive Health Care MSP.

8. WHO (2018). World Health Organization. Tuberculosis. [On-line].; 2018 [cited 2018 September 28. Available from: http://www.who.int/es/news-room/factsheets/detail/tuberculosis

9. Puc-Franco M, Caamal-Ley A and Vargas-González A. (2012). Social determinants related to tuberculosis treatment. Rev Biomed. 2012 February; 23 (113-120).

10. USAID (2000). United States Agency for International Development and Ministry of Health. Live tuberculosis In Socio-anthropological study of TB in high-risk areas. San Cosme, The Augustinian; 2000. p. 29. 
Arbitrada Interdisciplinaria de Ciencias de la Salud. SALUD Y VIDA

Volumen 3. Número 6. Año 3. Julio - Diciembre 2019

Hecho el depósito de Ley: FA2016000010

ISSN: 2610-8038

FUNDACIÓN KOINONIA (F.K).

Santa Ana de Coro, Venezuela.

Rosa del Rocío Pinargote Chancay; Yasmin Casrtillo Merino; Bryan Ramón Figueroa Merino; Geomayra Betzabeth Cedeño Chavarrria

(C2019 por los autores. Este artículo es de acceso abierto y distribuido según los términos y condiciones de la licencia Creative Commons Atribución-NoComercial-Compartirlgual 4.0 Internacional (CC BY-NC-SA 4.0)

(https://creativecommons.org/licenses/by-nc-sa/4.0/). 